\section{Going into resonance}

The explosive growth in the amount of information stored and processed in digital devices over the past 50 years has, in large part, been due to spectacular developments in techniques for fabricating nanostructured materials. Magnetic islands, stripes and layers can now be made with almost arbitrary precision, and technologies harnessing both their magnetic and electronic properties down to atomic length scales have emerged to give rise to an area of research that has become known as spintronics.

Accurately characterizing the properties of nanostructures that make up spintronic devices represents a formidable challenge, because conventional crystallographic approaches that work for bulk or powder samples become ineffective for systems of such reduced size and dimension. Their microscopic magnetic structure, for example, remained largely out of reach until the early 1980s.

In the mid-1980s, Doon Gibbs and colleagues at Brookhaven National Laboratory in the United States reported an intriguing development. Building on early theoretical work by Platzman and Tzoar, Gibbs and his colleagues showed that tuning the energy of $\mathrm{X}$-rays impinging on a thin holmium crystal so that they matched, or resonated with, the 'absorption edge' characteristic of a specific electronic binding energy, greatly enhanced the magnetic signal. Like many other rare-earth elements, the magnetic structure of holmium is characterized by a helical ordering of the magnetic moments, an arrangement

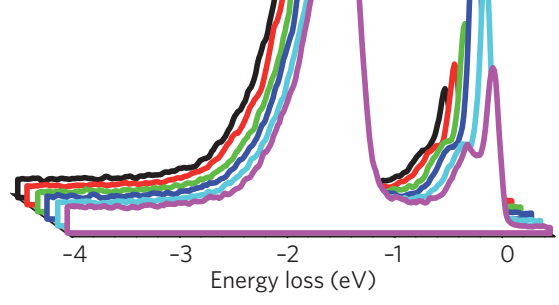

Resonant inelastic $\mathrm{X}$-ray scattering spectra for thin films of the cuprate superconductor $\mathrm{Nd}_{12} \mathrm{Ba}_{18} \mathrm{Cu}_{3} \mathrm{O}_{7}$. Figure reprinted with permission from G. Ghringhelli et al. Science 337, 821-825 (2012).

known as a spin spiral. Using the resonant $\mathrm{X}$-ray technique it was possible to map out this structure.

In effect, resonant magnetic X-ray diffraction combines diffraction with absorption spectroscopy. In addition to the magnetic moments, or spins, it is also sensitive to modulations in the charge and orbital ordering in a material, both of which are very difficult to observe using traditional scattering techniques. Moreover, the technique is element specific: the advent of second- and third-generation synchrotrons (Milestone 16) made it possible to selectively tune intense $\mathrm{X}$-ray beams at energies close to element absorption edges. This extreme sensitivity also had its drawbacks. For example, for energies at which air and sample absorption is very high, experiments must be done under vacuum and result in rather short penetration depths. But as the interest in nanoscale magnetic structures grew, the availability of a technique that seemed tailor-made for investigating magnetic and electronic surface and interface effects proved invaluable.

Along with the rapid enhancement of instrumental capabilities, resonant scattering techniques have continued to evolve over the past three decades. One especially significant development has been the recent rise of resonant inelastic $X$-ray scattering, a technique pioneered by Lucio Braicovich and Giacomo Ghiringhelli, among others, as an important tool for probing collective excitations in solids. Of course, excitations such as spin waves - the collective motion of the magnetic moments in a system - can also be measured in neutron scattering experiments (Milestone 8). But the sensitivity of the resonant approach also makes them measurable in very small volume samples and thin films. For example, a series of resonant inelastic X-ray scattering experiments performed on cuprate superconductors have already helped to significantly shape the discourse on their electronic structure and the mechanism for high-temperature superconductivity. Resonant magnetic $\mathrm{X}$-ray diffraction techniques look set to continue to profoundly influence our understanding of correlated states of matter in the future.

Andrea Taroni, Senior Editor, Nature Materials

ORIGINAL RESEARCH PAPERS Platzman, P. M. \& Tzoar, N . Magnetic scattering of $X$-rays from electrons in molecules and solids. Phys. Rev. B 2, 3556-3559 (1970) | de Bergevin, F. \& Brunel, M. Diffraction of $X$-rays by magnetic materials. I. General formulae and measurements on ferro- and ferrimagnetic compounds. Acta Crystallogr. A 37, 314-324 (1981)| Gibbs, D. etal. Magnetic X-ray scattering studies of holmium using synchrotron radiation. Phys. Rev. Lett. 55, 234-237 (1985) I Gibbs, D. etal. Polarization and resonance properties of magnetic X-ray scattering in holmium. Phys. Rev. Lett. 61, 1241-1244 (1988) | Ghringhelli, G. et al. Long-range incommensurate charge fluctuations in $(\mathrm{Y}, \mathrm{Nd}) \mathrm{Ba}_{2} \mathrm{Cu}_{3} \mathrm{O}_{6}$ Science 337, 821-825 (2012)

FURTHER READING Ament, L. J. P., van Veenendaal, M. Devereaux, T. P., Hill, J. P. \& van den Brink, J. Resonant inelastic $\mathrm{X}$-ray scattering studies of elementary excitations. Rev. Mod. Phys. 83, 705-767 (2011) | Fink, J., Schierle, E., Weschke, E. \& Geck, J. Resonant elastic soft X-ray scattering. Rep. Prog. Phys. 76, 056502 (2013) 